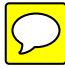

\title{
Sailing without wind sensor and other hardware and software innovations
}

\author{
Jan SLIWKA, Jeremy NICOLA, Remi COQUELIN, Francois BECKET DE \\ MEGILLE, Benoit CLEMENT, and Luc JAULIN
}

\begin{abstract}
In our paper, we propose a solution for a robotic sailboat which is adapted to long travel across the ocean. We tried to be as innovative as possible and stray out of the classical designs. In fact, robots often have different shapes from vehicles for mankind since first of all there are no human on board which means less constraints with regards to security, ergonomy or comfort. In this paper two innovations are presented. The first one addresses the main issue when navigating for a long time with a fully actuated sailing robot which is the energy used for guidance, navigation and control. One can use big solar panels to overcome that problem. Instead, we propose to use a wind vane self steering device. Our idea is to put this device on the bow which simplifies its design. This device regulates the trajectory of the boat relatively to wind direction. As a consequence, the wind sensor is not necessary. The second innovation is a strategy of navigation between waypoints. This paper will also present the prototype we used for testing those algorithms.
\end{abstract}

\section{Introduction}

Since many years unmanned surface vehicles (USVs) has been used for applications such as oceanography, harbour protection or military (as weapons or moving targets) [2]. USVs generally use propellers to move an get their energy from fossil fuels or solar power. It is only recently that USVs use sail as a mean of propulsion [1] and also became autonomous. The use of such vehicles is especially needed in oceanography [7][8][9] where there is a need for several months of data acqui-

Jan SLIWKA

ENSTA Bretagne, 2 rue Francois Verny, 29200 Brest, FRANCE, e-mail: jan.sliwka@enstabretagne.fr

Jeremy NICOLA

ENSTA Bretagne, 2 rue Francois Verny, 29200 Brest, FRANCE, e-mail: jeremy.nicola@enstabretagne.fr 
sition in a designed area. In fact the currently used buoys use to drift away very quickly from the data acquisition area and are often impossible to moor because of ocean's depth. Research in autonomous sailing boats was also stimulated by the MicroTransat challenge [3] which purpose is to cross the Atlantic ocean in full autonomy. Many autonomous sailing robots builders tend to make copies of existing sailboats for mankind installing the same set of sensors and replacing human actuation by electronic actuators. ENSTA-Bretagne robotic team has chosen to work on sailing robots capable of navigation for long periods. In order to reduce the energy consumption and to improve the robustness of the robot, we focus on reducing the number of sensors and actuators (especially the wind sensor) and develop new algorithms and/or mechanical designs to deal with that handicap. In this paper, we propose a solution for a robotic sailboat which addresses the main issue when navigating for a long time with a fully actuated sailing robot which is the energy used for guidance, navigation and control. One can use big solar panels to overcome that problem. Instead, we propose the use of a wind vane self steering device. This kind of device actually exists on real boats [4], but our approach is to install this device on the bow instead of putting it on the stern since it greatly simplifies its design. A mechanical design proposition will be provided for this device. Our first aim is to validate trajectory stabilization by the device. The final purpose is to sail using only the self steering device (without any rudder on the stern). Sailing using the selfsteering device enables navigation relatively to the wind. As a consequence, there is no need for the wind sensor. Simulations are provided for navigation maneuvers such as tacking and jibbing for both cases when the regulator is on the bow and the classical case when it is on the stern.

In this paper we also present algorithms for navigation between waypoints. There are many different methods which can be used for autonomous navigation [11][6]. Our algorithm allows the boat to navigate on straight lines between waypoints taking the drift of the boat into consideration. Simulations results are provided to prove the usefulness of the approach.

Finally, we will present our test prototype which is used for testing the concepts above. The design has been driven by the simplicity in construction.

\section{Sailing without wind sensor}

\subsection{Wind rudder regulator}

According to [4] the idea that a sailing boat might be able to steer itself using wind did not appear until the twentieth century. It might be basically because the crew was cheap and was doing the job perfectly. The first model of self steering wind vane was installed on a motorboat in 1936. It was connected to the rudder by lines. Nowadays, the wind vane self steering devices are also used on sailboats. We remarked that those devices were put on the stern. In fact, a boat can often be controlled single- 


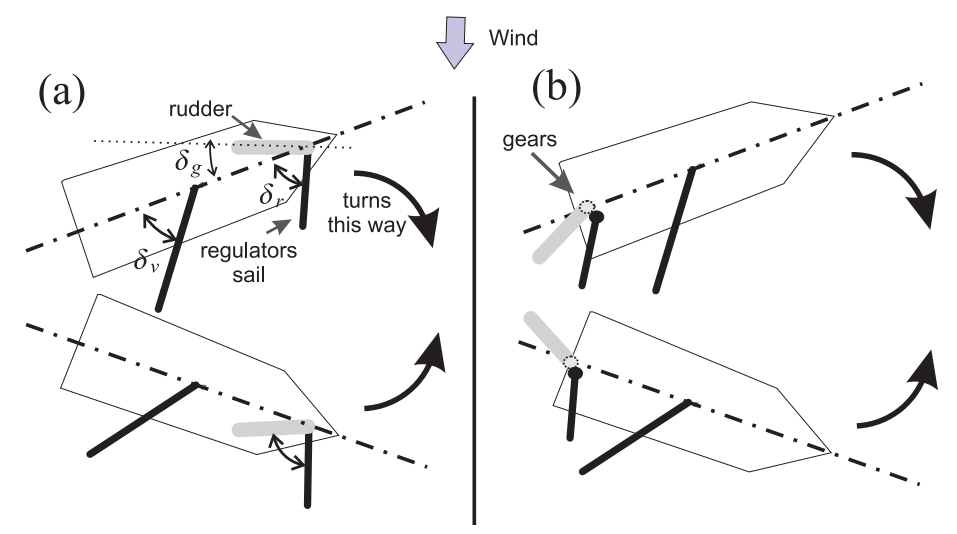

Fig. 1 Sub-Figure (b) shows a classical design of a wind vane self steering device when the rudder is on the stern. In this case there is a need to invert the rotation between the wind vane and the rudder using gears for example. Sub-Figure (a) shows the new simplified design where the rudder is on the front and the inversion of rotation is no longer necessary.

handedly by a human so naturally the wind vane, the rudder, sail lines are on the stern. Besides, the bow is often hardly accessible. Finally, the bow is subject to waves splash and more rough conditions. On the other hand, robot actuators can be dispatched everywhere on its body so that is when we came with the idea to put the self steering system on the bow. Figure 1 illustrate the difference between the bow and stern designs. The main difference is that there is no need for gears to invert the rotation between the wind vane and the rudder. We will remark later in the simulation part that those two designs are equivalent in terms of trajectory stabilization.

\subsection{Simulation}

\subsubsection{Equations}

Consider a dynamic system defined by the following evolution function

$$
\dot{\mathbf{x}}=\mathbf{f}(\mathbf{x}, \mathbf{u})
$$

$\mathbf{x}$ being the state of the system.

For the simulation, we use Euler approximation to estimate the state of the robot in real time as follows

$$
\mathbf{x}_{k+1}=\mathbf{f}\left(\mathbf{x}_{k}, \mathbf{u}_{k}\right) \mathbf{d t}+\mathbf{x}_{k}
$$




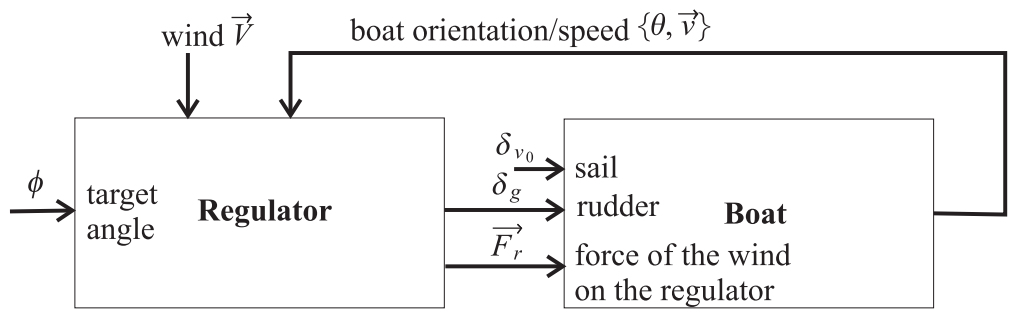

Fig. 2 This figure represents the block diagram corresponding to the system \{boat,wind vane regulator . The regulator will control the angle $\delta_{g}$ of the rudder of the boat and exerts a force $\mathbf{F}_{r}$ on the boat due to the drag of the wind vane. The regulator will be moved by the wind $(\mathbf{V})$. The angle between the rudder and the wind vane when the rudder is in zero position is determined by the variable $\phi$. The sail command is left constant.

$\mathbf{x}_{k}$ being the state of the system and $u_{k}$ being the command at time step $k$. For the simulation of the robot, we used the state equations from [5]. Figure 2 shows the block diagram of the system \{boat,wind vane regulator\}.

Denote by $\delta_{r}$ and $\delta_{g}$, respectively the angle between the wind vane and the axis of the boat, and the angle between the rudder and the axis of the boat as shown in Figure $1 . \delta_{v 0}$ is the sail command which will be left constant for the simulation. In fact, our prototype have an non actuated sail to add robustness. Denote by $\vec{V}$ the wind vector. Denote by $\phi$ the angle between the rudder and the wind vane when the rudder is in zero position. $\phi$ determines the target angle to be reached by the boat with respect to wind direction.

We made some assumptions in order to simplify the model. First we assumed the rudder is perfectly compensated i.e. the force due to the water current is applied directly on the axis of the rudder. As such, the wind vane (not hindered by the torque of the rudder) will align itself with the apparent wind unless the rudder is blocked by reaching its limit angle. We also assumed that the apparent wind is equal to the real wind. This approximation is valid for slow boats such as ours. We also neglected the dynamics of the sail and wind vane since their inertia is small with regards to the magnitude of forces generated by the wind.

When the rudder is not blocked i.e. $\delta_{g} \in\left[-\delta_{g_{\max }}, \delta_{g_{\max }}\right]$ we have,

$$
\phi=\delta_{r}-c \cdot \delta_{g}
$$

where $c=1$ corresponds to the case where the self steering mechanism is on the bow and $c=-1$ when it is on the stern.

The wind and the regulator are aligned thus

$$
\begin{aligned}
& \pi+\delta_{r}+\theta=\arg (\vec{V}) \\
& F_{r}=0
\end{aligned}
$$

As such

$$
\delta_{g}=c(\arg (\vec{V})-\pi-\theta-\phi)
$$




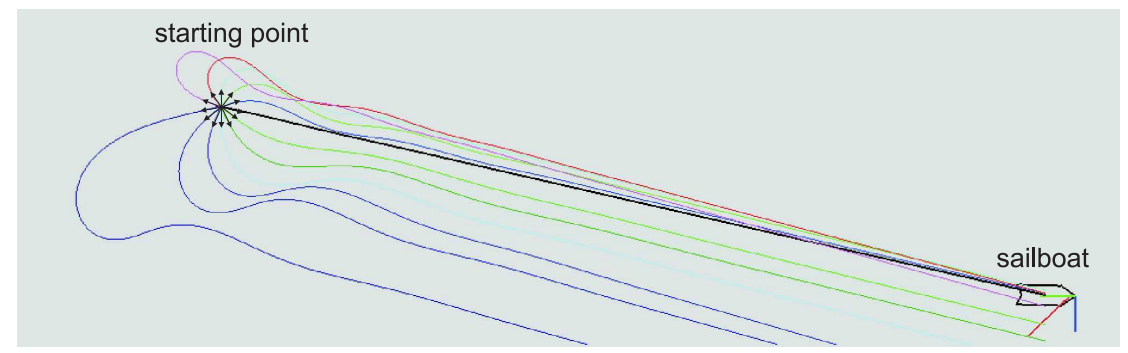

Fig. 3 We made SCILAB simulation. We launched the boat in several different directions. We observed that the boat is able to reach the desired course whatever the starting angle is which validates our approach. (simulation wise)

When the rudder reaches its limits, it becomes blocked. In this case, we have $\delta_{g}=-\delta_{g_{\max }}$ or $\delta_{g}=\delta_{g_{\max }}$ depending on the orientation of the boat relatively to the wind and the sign of $\phi$. In this case one can compute the force $F_{r}$ of the wind applied to the wind vane and consequently on the boat.

We have

$$
\delta_{r}=\phi+c \cdot \delta_{g}
$$

Consider $\delta_{r 0}$ the angle of the wind vane in the global workspace.

$$
\delta_{r 0}=\theta+\pi+\phi+c \cdot \delta_{g}
$$

As such

$$
F_{r}=F_{r \max } \operatorname{det}\left(\overrightarrow{u_{V}}, \overrightarrow{u_{\delta_{r 0}}}\right)
$$

where $F_{r \text { max }}$ is the force applied on the wind vane when it is perpendicular to the wind. $\overrightarrow{u_{V}}=\frac{\vec{V}}{\|\vec{V}\|}$ and $\overrightarrow{u_{\delta_{r 0}}}=\left(\cos \delta_{r 0}, \sin \delta_{r 0}\right)$.

\subsubsection{Result of stabilization}

Figure 3 represents the trajectories computed while starting the boat at different angles. We observed that the boat is able to reach the desired course whatever the starting condition is.

\subsubsection{Navigation using only the self steering device}

It is possible to navigate using only the self steering device by progressively changing the target angle $\phi$. Figure 4 shows the tacking and jibbing maneuvers for both case of the self steering device. In fact, the trajectories are the same. However, in reality, the big sail will hide the wind vane of the self steering device in some of 


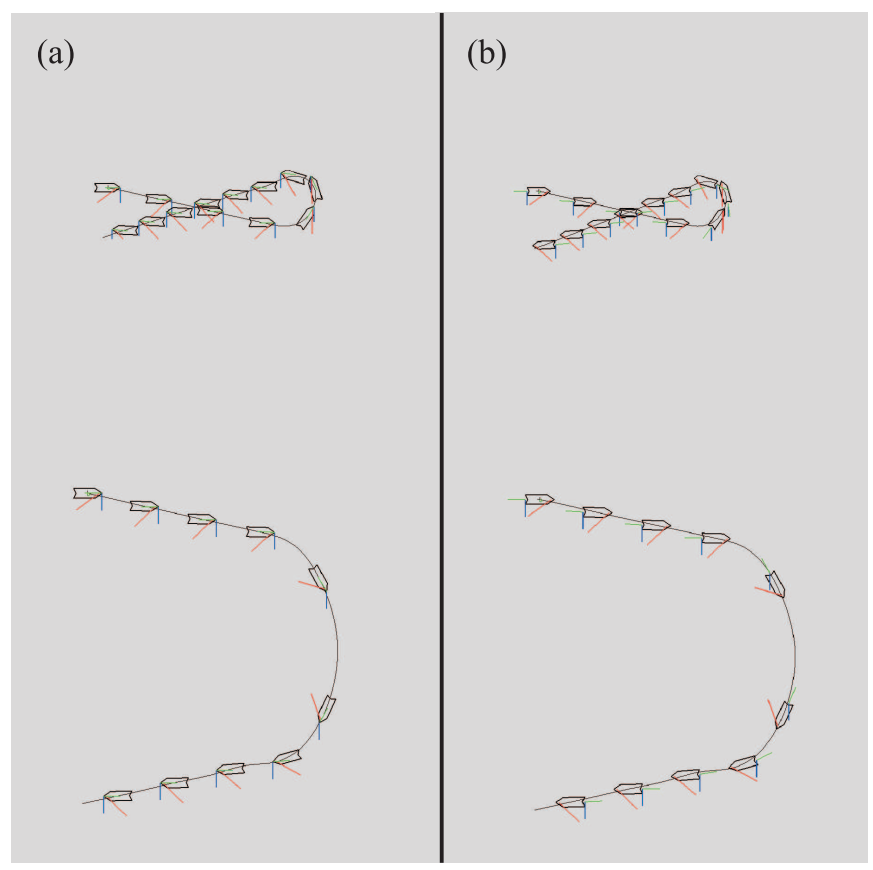

Fig. 4 Executing jibbing and tacking maneuvers using the self steering device (SCILAB simulation). Sub-Figure (a) corresponds to the case where the self steering device is on the bow. SubFigure (b) corresponds to the case when it is on the stern.

the cases. As a consequence, unless rising the wind vane above the big sail (or the opposite) the boat might have difficulties to jibe with a bow self steering device and to tack with a stern self steering device.

Note that it is necessary to have some speed to perform tacking or else the boat will be unable to cross the wind line. We are currently working on strategies to force the tacking meneuver when the speed of the boat is null. The idea is to block the rudder and use the wind vane to push the boat backwards. If the rudder is blocked in a suitable position the tacking maneuver will be accomplished.

\subsection{Mechanical design}

In this part we explain in more details the mechanical design of the bow wind vane self steering system. Installing the system on the bow simplifies the design of the system since there is no need for gears to invert the rotation of the wind vane and the rudder. As for the robustness, installing a mechanism bow put it under the action of the wave which might destroy it. However, in case of a bow self steering device the most fragile part, which is the wind vane, will align itself with the wind and the 


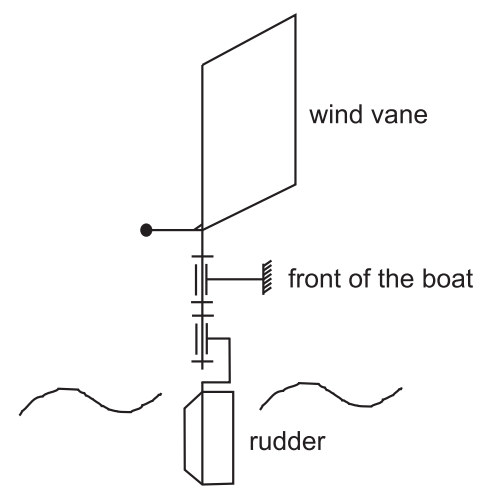

Fig. 5 The kinematic diagram of the bow wind vane self steering device

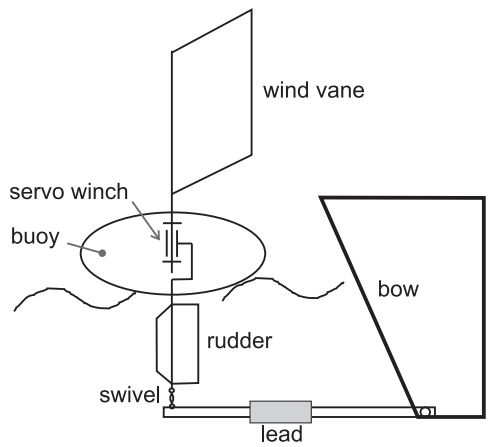

Fig. 6 Our front wind rudder regulator design.

waves reducing their impact on the structure. Figure 5 represents the overall design of the mechanism.

The mechanics is really simple. However, because the robot is small, the linkage has to have less friction than the one used on big boats since the applied forces are smaller. To overcome this problem, it is possible to use bearings but because of salt corrosion, one have to use special bearings such as plastic bearings with glass balls. We propose the following design of the bow self steering system using the fact that a round buoy rotates smoothly on sea water as seen in Figure 6.

Figure 7 represents our first attempt to construct the self steering device. The buoy is constructed using extruded polystyrene covered with fiberglass and resin for more robustness. A PVC sheet is sandwiched between two parts of the polystyrene. The PVC sheet supports a servowinch and a box for the electronics. 


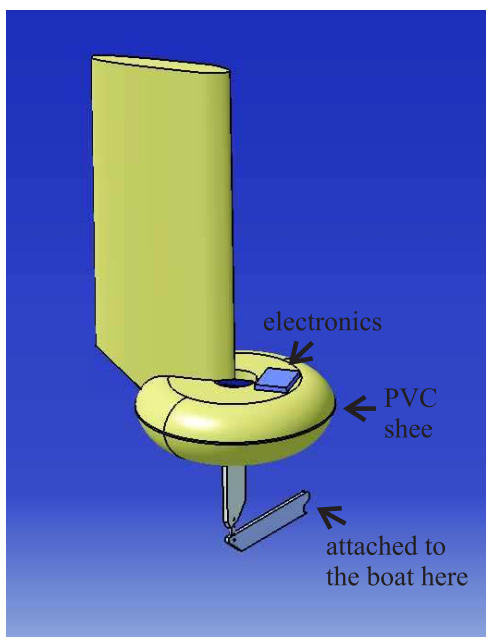

Fig. 7 The actual design of the first prototype using CATIA (computer assisted prototyping software)

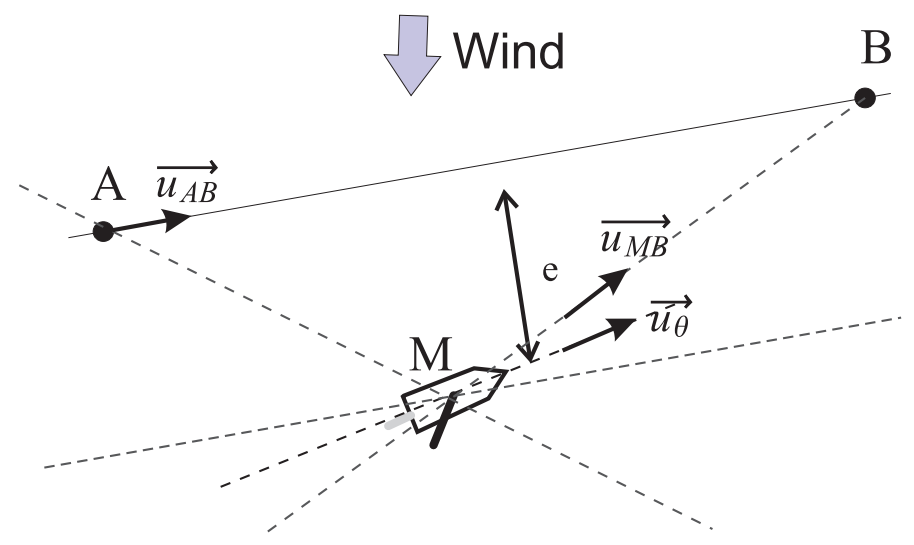

Fig. 8 This figure illustrates a sailboat navigating from a waypoint A to a waypoint B.

\section{Navigating in straight line}

We consider a robot boat navigating from a waypoint $A$ to waypoint $B$ as seen in Figure 8. If the waypoint $B$ is not upwind, the strategy is to try to regulate the boat such as it always targets waypoint $B$. Denote by $M=(x, y)$ the position of the robot and $B=\left(x_{b}, y_{b}\right)$ the position of the waypoint and $\theta$ the orientation of the robot (given by compass measurements for example). Consider the following vectors 


$$
\begin{aligned}
& \overrightarrow{u_{\theta}}=(\cos \theta, \sin \theta) \\
& \overrightarrow{u_{M B}}=\frac{\overrightarrow{M B}}{\|\overrightarrow{M B}\|} .
\end{aligned}
$$

Denote by $u \in[-1,1]$ the command associated to the rudder control. $u=0$ means a straight rudder, 1 rotated to the max position counterclockwise and -1 rotated to the max position clockwise. The command $u$ will be

$$
u=K * \operatorname{det}\left(\overrightarrow{u_{M B}}, \overrightarrow{u_{\theta}}\right) .
$$

This strategy works but due to the drift, the robot will be facing the wind when approaching the waypoint $B$ and eventually slow down (see trajectory (a) on Figure 9). We propose a hybrid approach which consists on following two different rules (and not one). The first rule is to follow the direction $\overrightarrow{A B}$. This rule is not sufficient to reach waypoint $B$ because of the drift (see trajectory (c) on Figure 9). The second rule is to minimize the distance $e$ between the boat and the line $(A B)$. As such, the boat will have a tendency to sail parallely to the line $(A B)$. Also, the boat will not loose speed arriving at the waypoint $B$.(see trajectory (c) on Figure 9)

Consider the vector

$$
\overrightarrow{u_{A B}}=\frac{\overrightarrow{A B}}{\|\overrightarrow{A B}\|} .
$$

The distance $e$ between the robot and the line $(A B)$ is expressed as

$$
e=\operatorname{det}\left(\overrightarrow{u_{A B}}, \overrightarrow{A M}\right) .
$$

The basic hybrid command $u$ can thus be defined by

$$
u=K_{1} * \operatorname{det}\left(\overrightarrow{u_{A} \vec{B}}, \overrightarrow{u_{\theta}}\right)+K_{2} * e .
$$

In practice there are some conditions so that this formula works better. For example, we started by regulating the angle of the boat first before caring about the distance $e\left(K_{2}=0\right.$ while $\operatorname{det}\left(\overrightarrow{u_{A B}}, \overrightarrow{u_{\theta}}\right)$ important). For more stability, when the boat is nearly on the line $(A B)$ we replace $K_{2} * e$ by $K_{2} * \arctan \left(K_{3} * e\right)$.

When it is possible to have a good estimation of the speed vector (which is not always the case especially if the speed of the robot is slow), we propose a command giving similar results to the last hybrid command. The idea is to regulate the boat such as its speed vector denoted by $\overrightarrow{u_{v}}$ points the waypoint $B$. The command becomes

$$
u=K * \operatorname{det}\left(\overrightarrow{u_{M B}}, \overrightarrow{u_{v}}\right) .
$$

Remark 1. Note that because of the drift $\overrightarrow{u_{v}} \neq \overrightarrow{u_{\theta}}$. 


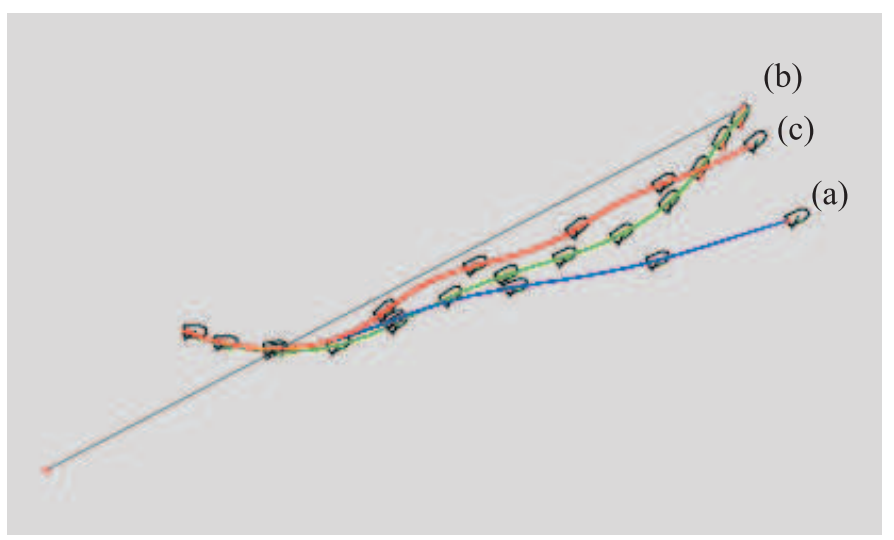

Fig. 9 Simulation results of three different algorithms.

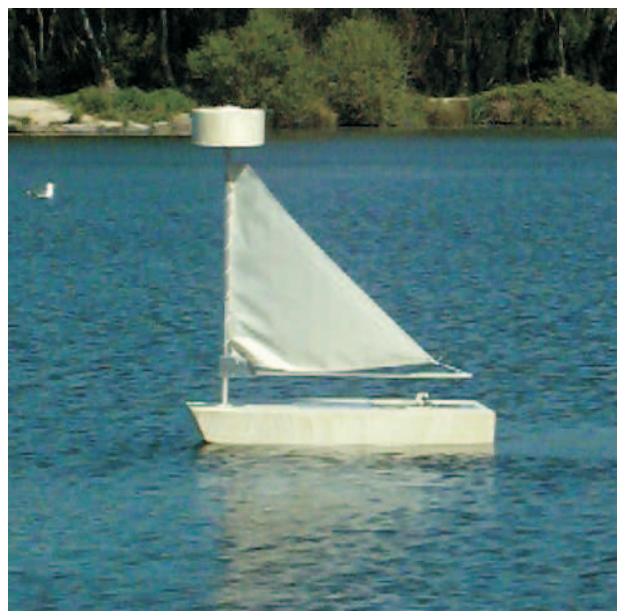

Fig. 10 The robot "L'improbable"

\section{Our prototype}

\subsection{Introduction}

In 2011, two prototypes were developed at ENSTA Bretagne. One is a copy of "Breizh Spirit" [10] and the other is "L'improbable" (see Figure 10) which is based on the "Optimist" type boat design (http: / / optiworld. org/). "L'improbable" is designed to try new solutions in terms of long period ocean navigation. 


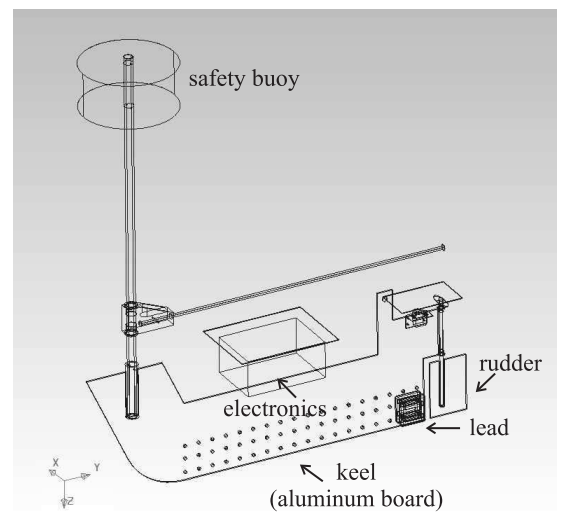

Fig. 11 The core board of "L'improbable" is an aluminum board which supports the mast, the rudder and the hull.

\subsection{Mechanical design of "L'improbable"}

The core of the boat is an aluminum board which acts as a keel and supports the mast as seen in Figure 7. The rudder is attached to the plaque as well. Surrounding the board there is an extruded polystyrene made hull. The hull was profiled using hot wire cutting technique which is widely used in RC plane model construction. The design core board + extruded polystyrene enables fast and robust prototyping of any type of boats. The polystyrene can be made more resistant by covering it with a fiberglass layer.

We decided not to actuate the sail to increase even more the robustness of the boat. The sail is thus set at a fixed angle (an empirical angle which works the best). However, even if we loose optimality, the preliminary tests on the Ty-Colo lake (see Figure 8) showed that the boat can navigate upwind. The navigation between waypoints as shown in section 3 has also been tested using the prototype.

\section{Conclusion}

In this paper we presented a possible solution to reduce electrical power consumption by replacing electronic boat heading correction (electronic autopilot) by a mechanical correction using a wind vane self steering device. We presented simulated results of heading stabilization and navigation maneuvers using the self steering device. Simulations look promising. The advantage of navigation using only the wind vane self steering device is that this navigation is relative to the direction of the wind. As a consequence the wind sensor is no longer necessary. In this paper we also presented few algorithms to navigate between waypoints. The real tests were performed using our recently build test platform presented in the last section. 


\section{References}

1. http://www.uovehicles.com.

2. V. Bertram. Unmanned surface vehicles, a survey. Skibsteknisk Selskab, Copenhagen (Denmark), 2008.

3. Y. Briere, F. Bastianelli, M. Gagneul, and P. Cormerais. Challenge microtransat. In CETSIS 2005, Nancy (France), 2007.

4. P. C. Forthmann. Self-steering under sail. Windpilot.

5. L. Jaulin. Modelisation et commande d'un bateau a voile. In CIFA2004 (Conference Internationale Francophone d'Automatique), In CDROM, Douz (Tunisie), 2004.

6. A. Schlaefer R. Bruder, B. Stender. Model sailboats as a testbed for artificial intelligence methods. In IRSC2009, pages 37-42, 2009.

7. C. Sauze and M. Neal. An autonomous sailing robot for ocean observation. In TAROS, pages 190-197, 2006.

8. C. Sauze and M. Neal. Design considerations for sailing robots performing long term autonomous oceanography. In IRSC 2008, pages 21-29, 2008.

9. C. Sauze and M. Neal. Ocean sampling and surveillance using autonomous sailboats. In IRSC 2008, pages 30-36, 2008

10. J. Sliwka, P. H. Reilhac, R. Leloup, P. Crepier, H. D. Malet, P. Sittaramane, F. L. Bars, K. Roncin, B. Aizier, and L. Jaulin. Autonomous robotic boat of ensieta. In IRSC 2009, Matosinhos (Portugal), 2009.

11. R. Stelzer, T. Proll, and R. John. Fuzzy logic control system for autonomous sailboats. In IEEE International Conference on Fuzzy Systems, 2007. 